\title{
Interface-dominated Growth of a Metastable Novel
}

\author{
Alloy Phase
}

\author{
Subhendu Sarkar, Alokmay Datta, Purushottam Chakraborty \\ Surface Physics Division, \\ Saha Institute of Nuclear Physics, \\ 1/AF Bidhannagar, Kolkata 700 064, India. \\ and \\ Biswarup Satpati \\ Institute of Physics, \\ Sachivalaya Marg, Bhubaneswar 751 005, India.
}

July 23, 2021

\begin{abstract}
A new $\mathrm{DO}_{23}$ metastable phase of $\mathrm{Cu}_{3} \mathrm{Au}$ is found to grow at the interfaces of $\mathrm{Au} / \mathrm{Cu}$ multilayers deposited by magnetron sputtering. The extent of formation of this novel alloy phase depends upon an optimal range of interfacial width primarily governed by the deposition wattage of the dc-magnetron used. Such interfacially confined growth is utilized to grow $\mathrm{a} \sim 300 \mathrm{~nm}$ thick $\mathrm{Au} / \mathrm{Cu}$ multilayer with thickness of each layer nearly equal to the optimal interfacial width which was obtained from secondary ion mass spectrometry (SIMS) data. This growth technique is observed to enhance the formation of the novel alloy phase to a considerable extent. SIMS depth profile also indicates that the mass fragment corresponding to $\mathrm{Cu}_{3} \mathrm{Au}$ occupies the whole film while x-ray diffraction (XRD) shows almost all the strong peaks belonging to the $\mathrm{DO}_{23}$ structure. High resolution cross-sectional transmission electron microscopy (HR-
\end{abstract}


XTEM) shows the near perfect growth of the individual layers and also the lattice image of the alloy phase in the interfacial region. Vacuum annealing of the alloy film and XRD studies indicate stabilization of the $D 0_{23}$ phase at $\sim 150^{\circ} \mathrm{C}$. The role of interfacial confinement, the interplay between interfacial strain and free energy and the hyperthermal species generated during the sputtering process are discussed. 


\section{Introduction}

In metallic multilayer systems the large free energies available at the interfaces can help to form interfacial alloys when these free energies are comparable to or larger than the enthalpy of formation of the probable alloy phases [1, 2. In many such systems the alloy formed is of a non-bulk, metastable nature [3, 4, 5]. For multilayers of immiscible metals there exists a positive mixing enthalpy that in effect acts as an energy barrier to mixing [6, 7]. On the other hand for miscible metallic systems the formation enthalpy for different metastable crystalline or ordered phases are mostly negative and very close to each other [8]. Interfacial alloy formation in multilayers have been reported to take place via solid state reaction [9, 10], mechanical alloying [11, 12, 13], ion beam irradiation [14, 15] etc. Especially in case of the immiscible systems, alloying is achieved via ion beam irradiation technique, which can supply adequate energy to overcome the energy barrier. Almost all of the metastable alloys reported for immiscible systems are amorphous in nature, the basic idea being the persistence of a random arrangement of atoms formed by forced mixing and subsequent rapid quenching of the supplied energy [16]. The probability of obtaining ordered alloy phases increases as we go over to the miscible systems. Local density approximation (LDA) calculations for $\mathrm{Au} / \mathrm{Cu}$ systems have shown that a number of ordered metastable phases have minima of formation enthalpy close to $L_{2}$, the ordered stable phase of the gold-copper alloy $\mathrm{Cu}_{3} \mathrm{Au}$ in bulk [8], and indeed the first indication of a metastable phase of $\mathrm{Cu}_{3} \mathrm{Au}$ was observed in a thermal study of the bulk alloy [17. Metastable alloys could be easier to form at $\mathrm{Au} / \mathrm{Cu}$ interfaces, and sputter deposition techniques, because of their high quench rate, can be more effective in achieving such a situation. Furthermore, hyperthermal atoms formed during the sputter deposition process can facilitate to modify or create new phases present at the interfaces [18, 19].

In view of the above facts, we had previously studied $(\mathrm{Au} / \mathrm{Cu})_{n},(\mathrm{n}=2,10)$ multilayers deposited on glass and Si (001) at ambient temperature by dc-magnetron sputtering [20]. Figure 1 summarizes the results obtained from that study. The nominal thickness of each layer was $\sim 50 \mathrm{~nm}$ (Figure 1a). Secondary ion mass spectrometry (SIMS) (Figure 1b), and

high-resolution cross-sectional transmission electron microscopy (HR-XTEM) studies showed 
that the multilayer periodicity and the crystallinity of the individual layers were excellent. The double peaks in the Au signal at the multilayer interfaces observed in the SIMS profile are due to the presence of oxygen trapped preferentially in the $\mathrm{Cu}$ layers. This observation is attributed to be due to the 'matrix effect' [21], a common phenomenon in SIMS. The crystallinity of the individual layers were also brought out by the fact that the layers were highly oriented in the $\langle 111\rangle$ direction as obtained from the x-ray diffraction (XRD) results (Figure 1c). But the most interesting result of the study [20] was that a $\mathrm{DO}_{23}$ phase of $\mathrm{Cu}_{3} \mathrm{Au}$ alloy (Figure 1d), a metastable ordered phase predicted from LDA calculations [8] formed only at the multilayer interfaces. The interfacial location of the alloy was confirmed from SIMS results (inset of Figure 1b) where the $\mathrm{Cu}_{2} \mathrm{Au}$ fragment of $\mathrm{Cu}_{3} \mathrm{Au}$ alloy was seen to be confined only across the interfacial region. This was the first experimental finding of $\mathrm{Cu}_{3} \mathrm{Au}$ in the $\mathrm{DO}_{23}$ phase rather than its bulk $\mathrm{L1}_{2}$ form (Figure 1d). It was also confirmed that formation of this novel phase was not a direct outcome of the deposition process but primarily due to confinement at the multilayer interfaces [20]. The major hurdle towards studying this phase in greater detail was practically due to the insufficiency in the amount of this alloy phase. Hence, a proper structural confirmation of the $\mathrm{DO}_{23}$ phase could not be completed in that case. We therefore planned to overcome this difficulty through enhancement of the formation of this metastable alloy utilizing its growth under interfacial confinement.

In this communication we present a novel, simple but effective method to obtain the $\mathrm{DO}_{23}$ phase of $\mathrm{Cu}_{3} \mathrm{Au}$ in sizable amounts. As $\mathrm{Au}-\mathrm{Cu}$ pair is one of the commonest pairs of miscible metals and as the method is based on optimizing the interfacial growth of the alloy, we believe that this method can be applicable to metastable interfacial alloys of miscible metals in general. We have used this method to grow $\mathrm{Cu}_{3} \mathrm{Au}$ alloy in the $\mathrm{DO}_{23}$ phase in considerable amounts in a $\sim 300 \mathrm{~nm}$ thick $\mathrm{Au} / \mathrm{Cu}$ multilayer and have been able to confirm its structure from the number of new peaks it provided in x-ray diffraction. Upon vacuum annealing, this alloy phase stabilizes at around $150^{\circ} \mathrm{C}$, consistent with the higher enthalpy minimum of $\mathrm{DO}_{23}$ compared to $\mathrm{L1}_{2}$, as found from LDA calculations [8]. 


\section{Experimental}

As a first and necessary step towards achieving the interface-dominated growth, we needed to estimate the optimal conditions favoring the growth of this metastable alloy phase. Towards this end, we deposited $(\mathrm{Cu} / \mathrm{Au})_{2}$ multilayers on glass at ambient temperature by dc-magnetron sputtering in a Pfeiffer PLS500 sputter-coating unit. The multilayers were grown on the substrates (kept at room temperature) by sequential deposition of $\mathrm{Au}$ and $\mathrm{Cu}$. During deposition the substrates attached to the sample holder were rotated to ensure uniform film growth. The geometry of the deposition chamber was such that the depositing atoms arrive at an angle normal to the substrate. The distance between the target and the substrate was $11.0 \mathrm{~cm}$. The base pressure was $3.4 \times 10^{-6}$ mbar and prior Ar (purity 99.99\%, BOC) flushing minimized impurities during deposition. The working pressure was in the range $3.5-5.0 \times 10^{-3}$ mbar. The nominal thickness for each layer was $\sim 50 \mathrm{~nm}$. The substrates were cleaned thoroughly with trichloroethylene and methanol. The Ar gas flow rate was $10 \mathrm{sccm}$ at the time of deposition. These parameters were identical to those employed in Ref. [20]. Three sets of samples were deposited at three different wattages viz., 13, 25 and 50 watts. SIMS studies were carried out using a QMS-based SIMS instrument (HIDEN Analytical Ltd, UK) with a high performance triple quadrupole filter and a $45^{\circ}$ electrostatic sector-field energy analyser [22]. Bombardment was done with cesium ions (1 $\mathrm{keV}, 50 \mathrm{nA}$ ) and secondary negative ions were detected during the measurement. X-ray diffraction (XRD) was done using a Philips X-ray diffractometer with a fixed anode-type $\mathrm{Cu} \mathrm{K} \mathrm{K}_{\alpha}$ source of wavelength $1.54 \AA$. The power of the source was $800 \mathrm{~W}$. The diffraction angle $2 \theta$ was varied from $10^{\circ}$ to $100^{\circ}$. The minimum step size $(2 \theta)$ was 10 mdeg. For cross-sectional TEM (XTEM) studies, samples were prepared using a wire saw (SBT 850), a lapping and polishing machine (SBT 910), a dimple grinder (Gatan 656) and a precision ion polishing system (Gatan 691). The samples were lapped and dimpled up to 120 and $40 \mu \mathrm{m}$, respectively. Ion polishing was done with Ar ions at $3 \mathrm{keV}$ up to electron transparency. The TEM measurements were carried out in a JEOL JEM-2010 transmission electron microscope operating at $200 \mathrm{keV}$. 


\section{Results and Discussion}

\subsection{Optimization of the growth parameters}

XRD spectra for samples deposited at the different wattages are shown in Figure 2a. The position of the (111) peak of the $\mathrm{Cu}_{3} \mathrm{Au}$ alloy in the $\mathrm{DO}_{23}$ phase is indicated by an arrow. The peak to background ratio of the alloy formed at different wattages is tabulated in table 1 . This ratio has been normalized with respect to that for the sample deposited at 50 watts. It is clear from the table that the alloy is better formed at 25 watts since its (111) peak intensity is the strongest at this wattage. Alloy formation is hindered for both higher and lower wattage conditions. SIMS results (Figure 2b), on the other hand, indicate that the interfacial width increases with the deposition wattage. This is quantitatively shown in table 1 The interfacial widths have been measured from the full width at half maximum (FWHM) of the individual alloy peaks located only at the interfaces. Higher deposition wattage indicates deposition at a higher energy, resulting in the broadening of the interface between two deposited layers. However, from this table and Figure 2a it is clear that the $\mathrm{DO}_{23}$ phase is well formed only when the interfacial width is $\sim 7.0 \mathrm{~nm}$.

It is expected that the formation of this interfacial alloy phase will be enhanced as the number of interfacial atoms as a fraction of the total number of atoms is increased [9, 23]. However, for our case this does not seem to be the deciding factor, since the above fraction, given by the interface width as a fraction of total thickness, monotonically increases with increase in deposition wattage. The deciding factor here is rather a sharply defined degree of confinement for which the particular alloy is best formed.

\subsection{Growth and characterizations of the interface-dominated alloy}

In view of the above findings, we planned to prepare a multilayer having identical interfacial confinement conditions but with individual layer thickness reduced to about the thickness of the optimum interface. Figure 3 summarizes our interface-dominated growth process and the results of SIMS and XRD studies on the grown film. We deposited $(\mathrm{Cu} / \mathrm{Au})_{20}$ multilayers on glass by dc-magnetron sputtering with identical growth conditions as that of our previous set of samples of two and ten bilayers as already discussed in the experimental section. 
The well-defined periodicity of the multilayer was confirmed from SIMS and XTEM studies. The individual layer thickness for the present multilayer was about $8.0 \mathrm{~nm}$ (Figure 3a). We believed that such low individual thickness would make up an interface-dominated multilayer stack thereby maintaining the proper strain so as to form the tetragonal alloy almost throughout the entire multilayer. SIMS depth profile (Figure 3b, top) of the Cu signal of the multilayer indicates that the stack has a well-defined periodicity with all the 20 layers distinctly separated. More important, however, is the fact that the $\mathrm{Cu}_{3} \mathrm{Au}$ alloy is present almost throughout the entire multilayer as evident from the SIMS depth profiles (Figure 3b, bottom). Thus it is clear that the grown multilayer is an interface-dominated structure.

Structural aspects of the multilayer were ascertained from HR-XTEM and XRD results. The near-crystalline growth of the individual layers are evident from HR-XTEM results as seen from Figure 4. The $f c c$ nature of the individual layers is evident from the FFT pattern (Figure 4d) of the marked region of the Au layer. XRD spectra Figure 3c), on the other hand, exhibited some extra peaks which were not present in that obtained from samples of our earlier study [20]. Of the different peaks obtained, only two peaks could be identified with $\mathrm{d}_{111}$ of both $\mathrm{Au}$ and $\mathrm{Cu}$ in their bulk $f c c$ phase. None of the other peaks obtained from the XRD spectra in the detected $2 \theta$ range could be matched with the bulk phases of $\mathrm{Au}$, $\mathrm{Cu}$ or any of their binary alloys. Most of the peaks can only be assigned to the $D 0_{23}$ phase of $\mathrm{Cu}_{3} \mathrm{Au}$. In our previous study, we had obtained only one peak (viz., (111)) of the $\mathrm{DO}_{23}$ phase of $\mathrm{Cu}_{3} \mathrm{Au}$ and that was expected to be a weak peak from structure factor calculations. Here we observe intense peaks of (006), (015) and (114) and a weak (112) peak in addition to the earlier (111) peak. The diffraction peaks were assigned using the CELREF Ver. 3.0 package. The lattice parameters $a$ and $c$, obtained for the $D 0_{23}$ phase are $0.3778 \mathrm{~nm}$ and $1.3962 \mathrm{~nm}$ respectively. HR-XTEM image of the interfacial region shows the lattice of the alloy phase (Figure 4b). The periodicity of the atomic arrangement becomes clearer from the autocorrelation (Figure $4 \mathrm{e}$ ) of the marked region at the interface. The non- $f c c$ nature of the lattice is also evident from the corresponding FFT pattern (Figure 4f). The dihedral angle estimated from the FFT is found to be $35.23^{\circ}$ which is quite close to $34.66^{\circ}$, the angle between the (015) and (114) planes of the $D 0_{23}$ phase. The calculated ratio $\mathrm{d}_{015} / \mathrm{d}_{114}(=1.058)$ for the $\mathrm{DO}_{23}$ phase also matches with that $(=1.0684)$ obtained from the FFT results. The $D 0_{23}$ 
phase of the $\mathrm{Cu}_{3} \mathrm{Au}$ alloy is now confirmed. To our knowledge, this is the first time that such a non-bulk alloy phase of gold and copper could be grown at ambient temperature in substantial amounts, although such a phase was predicted earlier from LDA calculations [8]. In addition to the $\mathrm{DO}_{23}$ phase, $\mathrm{Cu}_{3} \mathrm{Au}$ in the $L_{2}$ phase with a lattice constant of $0.3778 \mathrm{~nm}$ (quite different from its bulk value of $0.3749 \mathrm{~nm}$ ) was also found to exhibit its (111) peak. The analysis of the HR-XTEM image of the Au lattice and the $D 0_{23}$ phase shows that the (015) planes of the alloy phase grows parallel to the (220) planes of the Au layer. However, it is to be noted that due to insufficient TEM data we are unable to confirm this orientational assignment.

\subsection{Annealing of the novel alloy phase}

We then proceeded to see the thermal behaviour of this novel alloy phase. The samples were vacuum annealed ( $\sim 1 \times 10^{-5}$ mbar using a turbo-molecular pump, Pfeiffer Vacuum) for about two hours each, in a quartz tube within a Thermolyne tube furnace. The heating rate was $1^{\circ} \mathrm{C} / \mathrm{min}$. The alloy was annealed at $100^{\circ} \mathrm{C}, 125^{\circ} \mathrm{C}$ and $150^{\circ} \mathrm{C}$. Results (Figure 5a) show distinct changes in the XRD profile as the temperature increases. It is evident from the figure that the peaks corresponding to the $D 0_{23}$ phase tend to become more intense as the temperature approaches $150^{\circ} \mathrm{C}$. Also, with the increase of temperature we see the emergence and growth of the (111) peak of $\mathrm{CuAu}$. Figure 5b presents the results of annealing of the film quantitatively in a plot of the XRD peak intensities (normalized with respect to that of $\mathrm{Au}$ (111) at room temperature) versus temperature. The plots show three major trends as the temperature rises, (a) $\mathrm{Au}$ (111) and $\mathrm{Cu}$ (111) peaks grow weaker, (b) $\mathrm{CuAu}$ (111) peak grows stronger quite rapidly and (c) strong peaks of $\mathrm{DO}_{23} \mathrm{Cu}_{3} \mathrm{Au}$ namely, (114), (015) and (006), as well as the (111) peak of $\mathrm{L}_{2} \mathrm{Cu}_{3} \mathrm{Au}$ grow stronger at a slow but steady rate. Also the weak peaks, (111) and (112) of $\mathrm{L1}_{2} \mathrm{Cu}_{3} \mathrm{Au}$ grow weaker as the temperature rises. From these trends we can infer that as annealing temperature approaches $150^{\circ} \mathrm{C}$ : (a) $\mathrm{Au}$ and $\mathrm{Cu}$ in the multilayer convert to alloys, an inference supported by (b) the rapid growth of the $\mathrm{CuAu}$ alloy and (c) the steady growth of the metastable $\left(\mathrm{DO}_{23}\right)$ and stable $\left(\mathrm{L1}_{2}\right)$ forms of the $\mathrm{Cu}_{3} \mathrm{Au}$ alloy. The rapid growth of $\mathrm{CuAu}$ is expected as this alloy corresponds roughly to the atomic ratio of $\mathrm{Au}$ and $\mathrm{Cu}$ in the multilayer and would be the probable final state 
of the multilayer film upon annealing at higher temperature [24]. What is interesting is the 'stabilization' of the metastable alloy $\mathrm{DO}_{23}$ at $150^{\circ} \mathrm{C}$. The lattice parameter $a$, estimated from our XRD results is $0.3778 \mathrm{~nm}$. This remains unchanged upto $150^{\circ} \mathrm{C}$. The other lattice parameter $c$, varies from $1.3962 \mathrm{~nm}$ to $1.3952 \mathrm{~nm}$ in the $27^{\circ} \mathrm{C}-150^{\circ} \mathrm{C}$ temperature range.

Therefore, the direction of relaxation of this phase is along its $c$ direction, the direction of tetragonal distortion. It is to be noted that this stabilization or relaxation is taking place at about 240 degrees lower than the bulk $L 1_{2}$ phase stabilization temperature, indicating a shallower minimum in enthalpy as compared to the $L 1_{2}$ phase, consistent with the results of the LDA calculation [8]. Also such low temperature 'stabilization' of metastable phases can be caused by local attractive potentials that utilize the small extra movement caused by annealing to 'trap' more atoms thereby forming the alloy in the metastable phase. The considerable strain at the interfacial region caused by this local potential is indicated by the shift of the (111) peak of $L_{2} \mathrm{Cu}_{3} \mathrm{Au}$ from its bulk value. It is found that the lattice parameter $a$, has a value of $0.3778 \mathrm{~nm}$ upto $150^{\circ} \mathrm{C}$. Upon further annealing, this reduces very slowly. It is interesting to note in this context that the lattice parameter of the $L 1_{2}$ phase always matches (even beyond $150^{\circ} \mathrm{C}$ ) the lattice parameter $a$, of the $\mathrm{DO}_{23}$ phase indicating again that the relaxation occurs only along the $c$ axis. The loss in strength of the (111) and (112) peaks of the $\mathrm{DO}_{23}$ phase with annealing is again expected as these peaks would be forbidden for a stable and relaxed $\mathrm{DO}_{23}$ phase. Above $150^{\circ} \mathrm{C}, \mathrm{CuAu}$ formation becomes dominant and neither any new alloy phases nor any interesting development of the $D 0_{23}$ phase is observed. It is to be noted that peaks due to oxides of copper are absent throughout the annealing process.

\subsection{Factors affecting the alloy growth}

In order to have an idea on the formation mechanism of this new alloy phase, we take a closer look at the growth process. The deposition wattage used for the growth of the layers serves a two-fold purpose in this context.

(1) It determines the width of the interfacial region in the multilayer system.

(2) More importantly, it supplies the amount of energy required for the formation of the 
alloy phase.

The first point is very important because the alloy is obtained only at the interfaces of the sequentially deposited $\mathrm{Au} / \mathrm{Cu}$ system. We do not get this metastable phase when $\mathrm{Au}$ and $\mathrm{Cu}$ are co-sputtered under identical deposition conditions [20]. There we get the bulk $L 1_{2}$ phase of $\mathrm{Cu}_{3} \mathrm{Au}$. The tetragonal phase formed at the multilayer interfaces is obtained due to the requisite amount of strain (arising out of the interdiffusion of $\mathrm{Au}$ and $\mathrm{Cu}$ atoms for the particular interfacial width) maintained throughout the multilayer. It is to be borne in mind that the strain will have a gradient across the interface, which in turn is determined by the stoichiometric gradient of $\mathrm{Au}$ and $\mathrm{Cu}$ atoms at the interface. Earlier investigations reveal that an in-plane strain will produce a strain in the out-of-plane direction which eventually turns out to be a long period superlattice $\left(\mathrm{A}_{5} \mathrm{BAB}\right)$ direction along the $c$-axis in our case [8]. Minimization of the interfacial in-plane strain should bring the in-plane lattice constant ' $a$ ' to a value in between those of bulk $\mathrm{Cu}$ and $\mathrm{Au}$, as is observed from our annealing experiments.

It may be worth mentioning that the formation of this alloy phase in the present case is due to interfacial mixing that is determined by the deposition conditions and interdiffusion does not probably play a major role. However, with the increase of temperature interdiffusion has been found to occur across the interfaces of these multilayers [25]. Accordingly, in the temperature range of our study, diffusion takes place along defects and grain boundaries present in the system and consequently, the alloy formed is seen to persist even up to $150^{\circ} \mathrm{C}$. Alloying due to interdiffusion has been observed to dominate for $\mathrm{Cu} / \mathrm{Au}$ systems after about $250^{\circ} \mathrm{C}[25]$. However, we have noticed the formation of a very small amount of $\mathrm{CuAu}$ alloy to start from about $100^{\circ} \mathrm{C}$.

In order to understand the second point in more detail, we have to look at the magnetron sputtering phenomenon. The hyperthermal species (sputtered atoms and reflected plasma gas neutral atoms) formed during the deposition process play a significant role in the growth process in terms of the crystallinity, microstructure etc. of the films. Earlier works indicate that metastable phases can also be formed due to these species [26, 27]. In dc-sputtering deposition systems, the growing film is mainly bombarded by sputtered target atoms and reflected neutral plasma gas atoms. These hyperthermal species have their energies in the range $1-1000 \mathrm{eV}$. The intensity of the bombardment is determined by the growth conditions: 
the chamber pressure, deposition wattage, geometry of the deposition system, etc. The mean free path $(\lambda)$ of the Ar ions depends upon the working pressure $P$ and is given by [28]

$$
\lambda_{A r^{+}} \approx 1.1 / P
$$

which is $2.2 \mathrm{~cm}$ for our case. In order to have reflected neutrals, the mean free path of the Ar atoms should be greater than the cathode dark space whose length $d(\mathrm{~cm})$ is given by Child-Langmuir equation [29]

$$
d^{2}=8.6 \times 10^{-9} V^{3 / 2} / J
$$

where $V$ (in $\mathrm{V}$ ) is the dc voltage applied to the cathode and $J$ (in $\mathrm{A} / \mathrm{cm}^{2}$ ) is the ion-current density. Under 25 watts deposition condition $\mathrm{d}_{A u}=0.1803 \mathrm{~cm}$ and $\mathrm{d}_{C u}=0.1312 \mathrm{~cm}$. Thus it is evident that the Ar atoms easily surpass the cathode dark space. Now the reflection coefficient $(\rho)$ is given by the relation

$$
\rho=1-\frac{m}{M}
$$

where $m$ and $M$ are the masses of the incident ion (i.e. Ar) and the sputtered atom (i.e. Au or $\mathrm{Cu}$ ), respectively. It turns out that the reflection coefficient of Ar from Au target $\left(\rho_{A u}\right)$ is 0.7969 while that for $\mathrm{Cu}$ target $\left(\rho_{C u}\right)$ is 0.37 . Thus it is clear that majority of the reflected neutrals arise when Au sputtering occurs. According to Kaminsky [30] the maximum and minimum energy of the reflected species are given by the relations

$$
\begin{aligned}
& E_{r}^{\max }=\left(\frac{M-m}{M+m}\right) E \\
& E_{r}^{\text {min }}=\left(\frac{M-m}{M+m}\right)^{2} E
\end{aligned}
$$

where $E$ is the energy of the incident ion. The values calculated (for 25 watts deposition) using the above equations for $\mathrm{Au}$ and $\mathrm{Cu}$ gives $292 \mathrm{eV}$ and $83 \mathrm{eV}$ respectively for $E_{r}{ }^{\max }$ and $193 \mathrm{eV}$ and $19 \mathrm{eV}$ respectively for $E_{r}{ }^{\min }$. The $E_{r}{ }^{\max }$ and $E_{r}{ }^{\text {min }}$ values for Au are more sensitive to the change in deposition wattage in comparison to $\mathrm{Cu}$. These reflected neutrals then tend to thermalize as they travel towards the growing film. The extent of thermalization 
is given by the energy loss of the reflected atom as it passes through the sputtering gas and can be estimated from the relation [31]

$$
E_{\text {final }}=\left(E_{0}-k_{B} T_{G}\right) \exp \left[\frac{P D \sigma}{k_{B} T_{G}} \ln \left(E_{f} / E_{i}\right)\right]+k_{B} T_{G}
$$

where $E_{0}$ is the energy of the reflected atom as it leaves the target, $T_{G}$ is the sputtering gas temperature, $P$ is the sputtering gas pressure, $D$ is the target-substrate distance, $\sigma$ is the collision cross-section assuming hard core interactions and $E_{f} / E_{i}$ is the ratio of energies before and after a collision. Thus it follows from the above equation that for a particular sputtering gas and sputtered atom combination the pressure-distance product $(P D)$, which is $55 \mathrm{~Pa}-\mathrm{mm}$ for our case, determines the extent of thermalization. Therefore, the thermalized atoms will finally have energies in the region $250-350 \mathrm{eV} 32$ while the mean bombarding energy per arriving Ar atom may be $\sim 20-25 \mathrm{eV}$ [28] or even less. The last values have been estimated from Ref. [28] where thermalization was calculated for sputtered C atoms. The reflected Ar atoms will thermalize even more effectively due to equal mass. Moreover, it is important to remember that some phenomena such as transport efficiency, bombardment frequency, electronic excitation, ionization etc. were not considered in the calculations. Although our calculations overestimate the real experimental values, it can still be argued that the reflected neutrals have sufficient energy necessary to provide the enthalpy of formation of the new alloy phase at the interfaces. This takes place most effectively at about 25 watts. We argue that the deposited energy is low for deposition at 13 watts while it is far higher at 50 watts deposition condition. This is somewhat similar to our observations while we anneal the sample. At lower temperatures there is very little change of the phase. The phase stabilizes at about $150^{\circ} \mathrm{C}$. Beyond this temperature the structure breaks down and we tend to get an $\mathrm{CuAu}$ bulk alloy.

\section{Conclusion and Outlook}

We have shown that a metastable $\mathrm{DO}_{23}$ phase of $\mathrm{Cu}_{3} \mathrm{Au}$ can be grown in substantial amounts by dc-magnetron sputtering. Interfacial confinement primarily drives this alloy formation. Additionally, hyperthermal atoms present in the deposition chamber help in the interface- 
dominated growth of the novel alloy phase. Our annealing experiments reveal that the alloy tends to stabilize as the temperature is increased to about $150^{\circ} \mathrm{C}$ which is 240 degrees below the annealing temperature of the bulk $L 1_{2}$ phase of $\mathrm{Cu}_{3} \mathrm{Au}$.

Our method of interface-dominated alloy growth can, in principle, be used to grow metastable alloys to any desired amount thus enabling one to study the properties of such alloys in greater detail. The nature of the interface potential and the exact energetics of the arriving hyperthermal species need to be understood to better control the growth of the present and other metastable alloy phases. The presence of such alloys, including the hitherto unobserved $\mathrm{CuAu}_{2}$ alloy, has been predicted from the same LDA calculations [8] and may be indicated by the presence of new diffraction peaks that could not be assigned (Figure 3c) in the present work. Such studies are underway.

\section{References}

[1] V. Fournee, J. Ledieu, T. Cai and P. A. Thiel: Influence of strain in Ag on $\mathrm{Al}(111)$ and $\mathrm{Al}$ on $\mathrm{Ag}(100)$ thin film growth. Phys. Rev. B 67, 155401 (2003).

[2] A. Davies, Joseph A. Stroscio, D. T. Pierce, J. Unguris and R.J. Celotta: Observations of alloying in the growth of Cr on Fe(001). J. Magn. Magn. Mater. 165, 82 (1997).

[3] L. T. Kong, J. B. Liu, and B. X. Liu: Prediction of possible metastable alloy phases in an equilibrium immiscible Y-Mo system by ab initio calculation. J. Mater. Res. 17, $528(2002)$.

[4] Z. J. Zhang and B. X. Liu: Solid-state reaction to synthesize Ni-Mo metastable alloys. J. Appl. Phys. 76, 3351 (1994).

[5] G. Abadias, C. Jaouen, F. Martin, J. Pacaud, Ph. Djemia, and F. Ganot: Experimental evidence for the role of supersaturated interfacial alloys on the shear elastic softening of Ni/Mo superlattices. Phys. Rev. B 65, 212105 (2002).

[6] A. R. Yavari, P. J. Desre and T. Benameur: Mechanically driven alloying of immiscible elements. Phys. Rev. Lett. 68, 2235 (1992). 
[7] E. Ma, J. H. He and P. J. Schilling: Mechanical alloying of immiscible elements: Ag-Fe contrasted with Cu-Fe. Phys. Rev. B 55, 5542 (1997).

[8] L. G. Ferreira, V. Ozolins and A. Zunger: Fitting of accurate interatomic pair potentials for bulk metallic alloys using unrelaxed LDA energies. Phys. Rev. B 60, 1687 (1999).

[9] Y. G. Chen and B. X. Liu: Interface-driven solid-state alloying in an immiscible Cu-W system. J. Phys. D: Appl. Phys. 30, 1729 (1997).

[10] R. B. Schwarz and W. L. Johnson: Formation of an amorphous Alloy by solid-state reaction of the pure polycrystalline metals. Phys. Rev. Lett. 51, 415 (1983).

[11] J. Y. Huang, J. Z. Jiang, H. Yasuda and H. Mori: Kinetic process of mechanical alloying in $\mathrm{Fe}_{50} \mathrm{Cu}_{50}$. Phys. Rev. B 58, R11817 (1998).

[12] G. A. Dorofeev, E. P. Elsukov and A. L. Ulyanov: Mechanical alloying of immiscible elements in the FeMg system. Inorganic Materials 40, 690 (2004).

[13] E. Ma and M. Atzmon: Calorimetric evidence for polymorphous constraints on metastable Zr-Al phase formation by mechanical alloying. Phys. Rev. Lett. 67, 1126 (1991).

[14] J. Koike, P. R. Okamoto, L. E. Rehn and M. Meshii: Amorphization in $\mathrm{Zr}_{3} \mathrm{Al}$ irradiated with 1-MeV e ${ }^{-}$and $\mathrm{Kr}^{+}$. Metall. Trans. A 21, 1799 (1990).

[15] L. S. Hung, M. Nastasi, J. Gyulai and J. W. Mayer: Ion-induced amorphous and crystalline phase formation in $\mathrm{Al} / \mathrm{Ni}, \mathrm{Al} / \mathrm{Pd}$, and $\mathrm{Al} / \mathrm{Pt}$ thin films. Appl. Phys. Lett. 42, $672(1983)$.

[16] Jurgen Hafner, From Hamiltonian to Phase Diagrams, Springer Series in Solid State Sciences, Vol. 70, Springer-Verlag, Berlin (1987).

[17] P. Bardhan and J. B. Cohen: A structural study of the alloy $\mathrm{Cu}_{3} \mathrm{Au}$ above its critical temperature. Acta. Crystallogr. A 32, 597 (1976). 
[18] Luke Hanley and Susan B. Sinnott: The growth and modification of materials via ion-surface processing. Surf. Sci., 500, 500 (2002).

[19] X. W. Zhou and H. N. G. Wadley: Hyperthermal vapor deposition of copper: athermal and biased diffusion effects. Surf. Sci., 431, 42 (1999).

[20] Subhendu Sarkar, Alokmay Datta, Purushottam Chakraborty and Biswarup Satpati: Formation of a tetragonal $\mathrm{Cu}_{3} \mathrm{Au}$ alloy at gold/copper interfaces. Surf. Interface Anal., 35, 793 (2003).

[21] Subhendu Sarkar and Purushottam Chakraborty: Preferential oxygen-trapping in metallic multilayers: A SIMS perspective. J. Chin. Chem. Soc. 48, 521 (2001).

[22] Subhendu Sarkar, Purushottam Chakraborty and Hubert Gnaser: Energetics of $\mathrm{MCs}_{n}{ }^{+}$molecular ions emitted from Cs ${ }^{+}$irradiated surfaces. Phys. Rev. B, 70, 195427 (2004).

[23] Y. G. Chen and B. X. Liu: Alloy phases formed in immiscible Cu-Mo and Cu-W systems by multilayer-technique. Journal of Alloys and Compounds, 261, 217 (1997).

[24] Binary alloy phase diagrams, Eds., T. B. Massalski, H. Okamoto and T. R. Subramanian, $2^{\text {nd }}$ edn., American Society of Metals (1996).

[25] Peter Madakson and Joyce C. Liu: Interdiffusion and resistivity of $\mathrm{Cu} / \mathrm{Au}, \mathrm{Cu} / \mathrm{Co}$, $\mathrm{Co} / \mathrm{Au}$ and $\mathrm{Cu} / \mathrm{Co} / \mathrm{Au}$ thin films at 25-550 C. J. Appl. Phys. 68, 2121 (1990).

[26] Y. Lifshitz, S. R. Kasi, J. W. Rabalais and W. Eckstein: Subplantation model for film growth from hyperthermal species. Phys. Rev. B 41, 10468 (1990).

[27] Y. Lifshitz, S. R. Kasi and J. W. Rabalais: Subplantation model for film growth from hyperthermal species: Application to diamond. Phys. Rev. Lett. 62, 1290 (1989).

[28] L. G. Jacobsohn and F. L. Freire, Jr.: Influence of the plasma pressure on the microstructure and on the optical and mechanical properties of amorphous carbon films deposited by direct current magnetron sputtering. J. Vac. Sci. Technol. A 17, 2841 (1999). 
[29] I. Langmuir: The effect of space charge and residual gases on thermionic currents in high vacuum. Phys. Rev. 2, 450 (1913).

[30] Atomic and ionic impact phenomenon, M. Kaminsky, Springer-Verlag, Berlin (1965).

[31] K. Meyer, I. K. Schuller and C. M. Falco: Thermalization of sputtered atoms. J. Appl. Phys. 52, 5803 (1981).

[32] R. E. Somekh: The thermalization of energetic atoms during the sputtering process. J. Vac. Sci. Technol. A 2, 1285 (1984). 
Table 1: Intensity of $\mathrm{DO}_{23} \mathrm{Cu}_{3} \mathrm{Au}$ (111) peak (peak to background ratio) and interfacial widths of films deposited under different wattages.

\begin{tabular}{|c|c|c|}
\hline $\begin{array}{c}\text { Deposition wattage } \\
\text { (watts) }\end{array}$ & $\begin{array}{c}\mathrm{Cu}_{3} \mathrm{Au} \text { (111) Intensity* } \\
\text { (from XRD) }\end{array}$ & $\begin{array}{c}\text { Interfacial width } \\
\text { (from SIMS) }\end{array}$ \\
\hline 13 & 1.103 & $6.0 \mathrm{~nm}$ \\
\hline 25 & 1.203 & $6.8 \mathrm{~nm}$ \\
\hline 50 & 1.000 & $8.0 \mathrm{~nm}$ \\
\hline
\end{tabular}

*Relative to intensity at 50 watts deposition power. 


\section{Figure Captions}

Figure 1.: (a) Cross-sectional TEM image of the $(\mathrm{Cu} / \mathrm{Au})_{10} / \mathrm{Si}$ multilayer, (b) SIMS depth profiles showing $\mathrm{Au}, \mathrm{Cu}$ and $\mathrm{Si}$ signals in a $(\mathrm{Au} / \mathrm{Cu})_{2} / \mathrm{Si}$ film; Inset: SIMS depth profiles showing $\mathrm{Cu}$ and $\mathrm{Cu}_{2} \mathrm{Au}$ alloy fragment signals in the same film, (c) XRD spectrum of the $(\mathrm{Au} / \mathrm{Cu})_{10} /$ glass multilayer and $(\mathrm{d})$ Crystal structures of $L 1_{2}$ and $D O_{23}$ structures.

Figure 2.: (a) XRD spectra of a Au-capped $(\mathrm{Cu} / \mathrm{Au})_{2} /$ glass multilayer deposited at (a) 13 watts, (b) 25 watts and (c) 50 watts. (b) SIMS depth profiles showing Au and Cu signals of a Au-capped $(\mathrm{Cu} / \mathrm{Au})_{2} /$ glass multilayer deposited at (a) 13 watts, (b) 25 watts and (c) 50 watts. The large humps in the Au signal are due to matrix effect [21].

Figure 3.: (a) Cross-sectional TEM image of the $(\mathrm{Cu} / \mathrm{Au})_{20} / \mathrm{Si}$ multilayer, (b) Top: SIMS depth profile showing $\mathrm{Cu}$ signal of a Au-capped $(\mathrm{Cu} / \mathrm{Au})_{20} /$ glass multilayer; Bottom: SIMS depth profiles showing $\mathrm{Cu}$ and $\mathrm{Cu}_{2} \mathrm{Au}$ alloy fragment signals up to 7 layers of the same multilayer and (c) XRD spectrum of the Au-capped $(\mathrm{Cu} / \mathrm{Au})_{20} /$ glass multilayer.

Figure 4.: (a) HR-XTEM image of a Au layer showing the well-ordered lattice planes, (b) HR-XTEM image of an interfacial region of the multilayer, (c) Autocorrelation of the marked region of (a), (d) FFT of the marked region of (a), (e) Autocorrelation of the marked region of (b), (f) FFT of the marked region of (b).

Figure 5.: (a) XRD results of annealing at different temperatures of the Au-capped $(\mathrm{Cu} / \mathrm{Au})_{20} /$ glass

multilayer, (b) Intensity of the peaks at different temperatures of the $\mathrm{Au}$-capped $(\mathrm{Cu} / \mathrm{Au})_{20} / \mathrm{glass}$ multilayer. 
This figure "figure1.png" is available in "png" format from: http://arxiv.org/ps/cond-mat/0506427v1 
This figure "figure2.png" is available in "png" format from: http://arxiv.org/ps/cond-mat/0506427v1 
This figure "figure3.png" is available in "png" format from: http://arxiv.org/ps/cond-mat/0506427v1 
This figure "figure4.png" is available in "png" format from: http://arxiv.org/ps/cond-mat/0506427v1 
This figure "figure5.png" is available in "png" format from: http://arxiv.org/ps/cond-mat/0506427v1 